\title{
Diagnostic Accuracy of Transcranial Doppler for Brain Death Confirmation: Systematic Review and Meta-Analysis
}

\author{
(D).J. Chang, (D) G. Tsivgoulis, (D)A.H. Katsanos, (D) M.D. Malkoff, and (D)A.V. Alexandrov
} $O=$

\begin{abstract}
BACKGROUND AND PURPOSE: Transcranial Doppler is a useful ancillary test for brain death confirmation because it is safe, noninvasive, and done at the bedside. Transcranial Doppler confirms brain death by evaluating cerebral circulatory arrest. Case series studies have generally reported good correlations between transcranial Doppler confirmation of cerebral circulatory arrest and clinical confirmation of brain death. The purpose of this study is to evaluate the utility of transcranial Doppler as an ancillary test in brain death confirmation.
\end{abstract}

MATERIALS AND METHODS: We conducted a systematic review of the literature and a diagnostic test accuracy meta-analysis to compare the sensitivity and specificity of transcranial Doppler confirmation of cerebral circulatory arrest, by using clinical confirmation of brain death as the criterion standard.

RESULTS: We identified 22 eligible studies (1671 patients total), dating from 1987 to 2014. Pooled sensitivity and specificity estimates from 12 study protocols that reported data for the calculation of both values were $0.90(95 \% \mathrm{Cl}, 0.87-0.92)$ and $0.98(95 \% \mathrm{Cl}, 0.96-0.99)$, respectively. Between-study differences in the diagnostic performance of transcranial Doppler were found for both sensitivity $\left(I^{2}=76 \%\right.$; $P<.001)$ and specificity $\left(I^{2}=74.3 \% ; P<.001\right)$. The threshold effect was not significant (Spearman $\left.r=-0.173 ; P=.612\right)$. The area under the curve with the corresponding standard error (SE) was $0.964 \pm 0.018$, while index $Q$ test \pm SE was estimated at $0.910 \pm 0.028$.

CONCLUSIONS: The results of this meta-analysis suggest that transcranial Doppler is a highly accurate ancillary test for brain death confirmation. However, transcranial Doppler evaluates cerebral circulatory arrest rather than brain stem function, and this limitation needs to be taken into account when interpreting the results of this meta-analysis.

ABBREVIATIONS: $C C A$ = cerebral circulatory arrest; $Q^{*}=$ index $\mathrm{Q}$ test; $\mathrm{QUADAS}=$ Quality Assessment of Diagnostic Accuracy Studies; sROC $=$ summary receiver operating curve; $\mathrm{TCD}=$ transcranial $\mathrm{Dopple} ; \mathrm{SE}=$ standard error

$\mathrm{T}$ he concept and irreversibility of brain death have evolved in the past 50 years, further differentiating it from "irreversible coma" as initially described in $1968 .{ }^{1}$ Today, the clinical examination, apnea test, etiology, and ascertainment of irreversibility; radiologic confirmation of a structural lesion; and elimination of

Received May 4, 2015; accepted after revision July 11.

From the Department of Neurology (J.J.C., G.T., M.D.M., A.V.A.), University of Tennessee Health Science Center, Memphis, Tennessee; Second Department of Neurology (G.T., A.H.K.), Attikon University Hospital, School of Medicine, University of Athens, Athens, Greece; International Clinical Research Center (G.T.), St. Anne's University Hospital in Brno, Czech Republic; and Department of Neurology (A.H.K.), University of Ioannina, School of Medicine, loannina, Epirus, Greece.

Please address correspondence to Jason Chang, MD, Department of Neurology, University of Tennessee Health Science Center, 1325 Eastmoreland, Suite 460, Memphis, TN 38104; e-mail: jjwchang@hotmail.com

- Indicates open access to non-subscribers at www.ajnr.org

$\equiv$ Indicates article with supplemental on-line appendix and table.

Mvidence-Based Medicine Level 2.

http://dx.doi.org/10.3174/ajnr.A4548 confounding laboratory tests remain the criterion standard for brain death diagnosis. ${ }^{2}$

Ancillary testing for brain death confirmation remains controversial. The latest guidelines from the American Academy of Neurology and American Academy of Pediatrics report insufficient evidence for determining brain death with ancillary tests. ${ }^{3,4}$ However, ancillary tests remain essential in brain death confirmation when clinical instability prevents safe use of an apnea test ${ }^{5,6}$ or barbiturate therapy or hypothermia precludes proper brain death confirmation. ${ }^{7}$ In such circumstances, ancillary testing may complement criterion standard testing and confirm brain death. Transcranial Doppler (TCD) is useful for ancillary brain death confirmation because it is safe and noninvasive. TCD confirms brain death by evaluating cerebral circulatory arrest (CCA), which has distinctive flow patterns: oscillatory flow representing reversal of diastolic flow and systolic spikes representing lack of net forward flow.

Case reports and case series have generally reported good correlations between TCD confirmation of CCA and clinical confir- 
mation of brain death, with sensitivities ranging from $70.5 \%$ to $100 \%$. Most recently, a meta-analysis of 10 studies demonstrated TCD having a sensitivity of $89 \%$ and specificity of $99 \%$ compared with the criterion standard clinical confirmation of brain death. ${ }^{8}$ However, this review omitted key methodologies, including estimation of publication bias and the relative strength of each study, and did not adopt Quality Assessment of Diagnostic Accuracy Studies (QUADAS) methodology that standardizes quality assessment and diagnostic accuracy of individual studies in systematic reviews. ${ }^{9}$

In view of these former considerations, we conducted a systematic review of the literature and a meta-analysis adopting the Preferred Reporting Items for Systematic Reviews and MetaAnalyses guidelines ${ }^{10}$ and by using QUADAS methodology ${ }^{9}$ for quality assessment of included studies. The aim of this meta-analysis was to compare the sensitivity and specificity of TCD confirmation of CCA with clinical confirmation of brain death.

\section{MATERIALS AND METHODS}

\section{Data Sources and Study Selection}

We searched PubMed, Scopus, and the Cochrane Central data bases for all published studies by using the terms "transcranial Doppler," "cerebral circulatory arrest," and "brain death." Three reviewers (J.J.C., G.T., and A.H.K.) examined the references independently to exclude duplicates. The last literature search was conducted on October 16, 2014. Relevant review articles were examined to identify those that might have been missed in the data base search. We imposed no language limitations on the literature search. Case reports, studies not offering a comparison criterion standard clinical examination, and studies consisting of an exclusively pediatric population or primarily focusing on TCD analysis in populations with structural defects of the cranium were excluded.

\section{Study Eligibility}

All retrieved studies were independently examined by 3 reviewers (J.J.C., G.T., and A.H.K.) to determine overall eligibility. Prospectively collected, retrospective, and case series were included. Inclusion criteria for studies were as follows: 1) clinical confirmation of brain death serving as a criterion standard; 2) insonation window that included a transtemporal, suboccipital, or transorbital approach; 3) studies in which quantitative numbers could be extrapolated to calculate sensitivities and specificities; and 4) ages ranging from neonate to 100 years of age while excluding studies focused solely on pediatric populations.

\section{Data Extraction}

The following information from each study was extracted by the 3 investigators independently: true-positives, false-negatives, truenegatives, false-positives, etiology of neurologic injury, and window used for insonation. When identifying the accuracy of TCD waveforms for sensitivity and specificity analysis, we used the following rules: First, waveforms that could not be obtained through the necessary bone windows on TCD were marked as a falsenegative result, ultimately lowering the sensitivity for the study and, in some cases, resulting in a lower sensitivity than that reported by the article. Second, for serial TCD examinations, sensi- tivity was calculated by using the last TCD examination completed because this would have been closest to CCA and would yield the highest sensitivity.

As recommended by the Cochrane Collaboration (http:// www.cochrane.org/), the QUADAS-2 tool (http://www.bris.ac.uk/ quadas/quadas-2/) was used to assess the risk of bias of each primary study that reported both sensitivity and specificity measures. ${ }^{9,11}$ The 3 reviewers independently evaluated QUADAS- 2 items, and conflicts were resolved by consensus.

\section{Data Synthesis and Statistical Analysis}

Details regarding data synthesis and statistical analyses are provided in the On-line Appendix. In brief, sensitivity and specificity were calculated for individual studies, and a random-effects analysis model (DerSimonian and Laird) was used for the estimation of both pooled sensitivity and specificity.

Heterogeneity between studies was assessed by the Cochran Q test and $\mathrm{I}^{2}$ statistic. Summary receiver operating curves were also constructed by using the random-effects model. The area under the curve and point of the curve where sensitivity equals specificity index $\mathrm{Q}$ test were used to assess and summarize the discriminating ability of the summary receiver operating curve (sROC).

\section{RESULTS}

\section{Study Selection and Study Characteristics}

We searched PubMed and Scopus data bases, initially identifying 310 and 376 studies, respectively, that dated from 1987 to 2014. After removing duplicates, we screened the titles and abstracts from the remaining 455 studies, yielding 36 potentially eligible studies for the meta-analysis. The Cochrane Central data base search retrieved no additional studies. Full-text versions of these 36 studies were obtained, and 14 studies were excluded for the following reasons: 1) They did not offer a comparison criterion standard clinical examination, ${ }^{12-14}$ 2) the exact number of patients could not be extrapolated from the methodology or results for analysis, ${ }^{15,16} 3$ ) the study consisted of an exclusively pediatric population, ${ }^{17-20} 4$ ) the article was a single-patient case report, ${ }^{21-23}$ or 5) the study primarily focused on TCD analysis in populations with structural defects of the cranium (ie, patients with external ventricular devices or postdecompressive hemicraniectomy). ${ }^{24,25}$

In the final presentation of the literature search results, there was no conflict or disagreement between 2 reviewers (J.J.C. and G.T.), and the 22 studies that met the protocol inclusion criteria of the study were included in the present systematic review (Fig 1). The characteristics of the included studies, ${ }^{26-47}$ comprising 1596 total patients, are shown in the On-line Table.

\section{Quality Assessment}

The quality assessment of included studies by using QUADAS- 2 is presented in the Table. High bias was introduced in all 22 studies when using the risk of bias/index test asking the question "could the conduct or interpretation of the index test have introduced bias?" because TCDs were always performed after confirmation of clinical brain death. Risk of bias/patient selection was introduced in 4 studies because patients were excluded due to either TCD windows being unobtainable $\mathrm{e}^{27}$ or, by study design only, a portion of the patients who were clinically brain dead underwent 


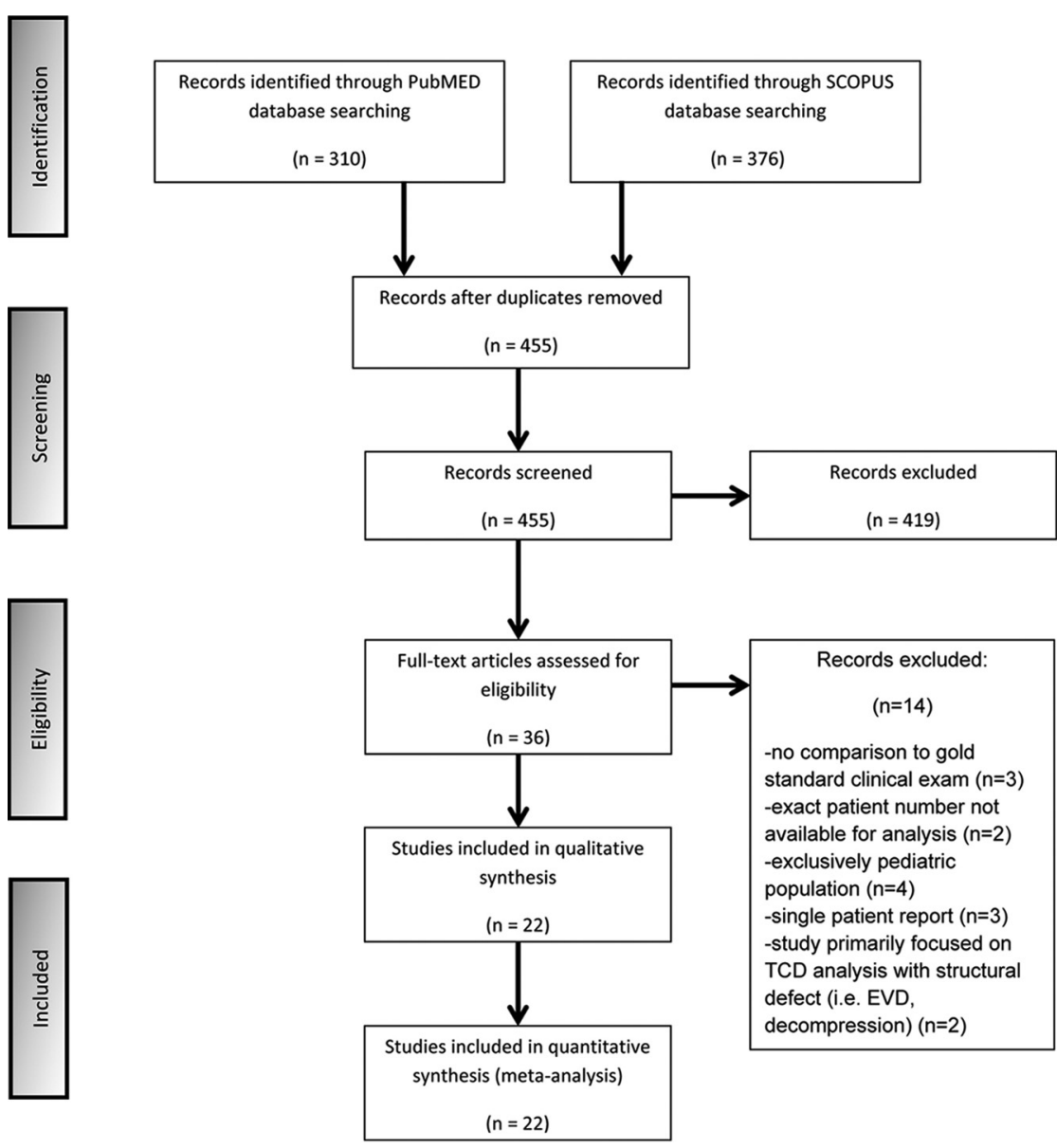

FIG 1. Flow chart diagram presenting the selection of eligible studies. EVD indicates external ventricular drain.

TCDs. ${ }^{34,38,43}$ High bias was introduced in risk of bias/flow and timing in 3 studies because patients were excluded from analysis due to TCD waveforms being unobtainable despite vasopressor use, ${ }^{28}$ bone windows or clinical brain death not being determined, ${ }^{30}$ and clinical brain death and even ancillary tests not being performed because of severe clinical instability. ${ }^{41}$ Unclear bias was introduced in applicability concerns/patient selection in 6 studies because pediatric patients were included in the analysis. ${ }^{33,34,37,42,45,47}$ Finally, high bias was introduced in 1 study in applicability concerns/index test because in addition to the standard TCD transtemporal window, contrast-induced TCD was used. ${ }^{26}$ No study had $>2$ high risks of bias noted.

\section{Data Synthesis and Statistical Analysis}

The sensitivity and specificity values, with the corresponding 95\% CIs, were calculated for all included studies. However, overall sensitivity and specificity estimates were pooled from 12 of the 22 study protocols because only these studies reported data for the calculation of both sensitivity and specificity values (Fig 2).

In the pooled analysis of 859 patients ( $56.1 \%$ with clinically confirmed brain death), overall sensitivity was 0.90 (95\% CI, 0.87-0.92; Fig 3A) and overall specificity was 0.98 (95\% CI, 0.96-0.99; Fig 3B). Between-study differences in the diagnostic performance of TCDs were found for both sensitivity $\left(\mathrm{I}^{2}=\right.$
76\%, $P<.001$, Fig $3 A)$ and specificity $\left(\mathrm{I}^{2}=74.3 \%, P<.001\right.$, Fig $\left.3 B\right)$. The threshold effect was not significant (Spearman $r=-0.173, P=.612$ ). The sROC analysis, displaying the individual studies that reported data for the calculation of both sensitivity and specificity and the sROC curve (with its corresponding 95\% CI), is presented in Fig 4. The area under the curve of sROC with the corresponding standard error (SE) was $0.964 \pm 0.018$, while the $\mathrm{Q}^{\star} \pm \mathrm{SE}$ was estimated at $0.910 \pm 0.028$.

\section{DISCUSSION}

This study provides the most comprehensive review evaluating the sensitivity and specificity of TCD in confirming brain death. The results of this metaanalysis suggest that TCD is a highly accurate ancillary test for brain death confirmation with a sensitivity and specificity of $90 \%$ and $98 \%$, respectively. Moreover, the area under the curve of sROC was estimated at 0.964 , underscoring the very satisfactory diagnostic yield of TCD for diagnosing CCA.

The findings of this meta-analysis are roughly in line with the prior meta-analysis of Monteiro et al, ${ }^{8}$ with a sensitivity of $89 \%$ and specificity of $99 \%$ when using TCD for brain death confirmation. In our meta-analysis, we included all 12 studies used in that article and 10 additional studies. We also improved on the study design by systematically evaluating potential sources of bias and the relative strengths of each study. Moreover, sROC analyses were not performed in the study of Monteiro et al, while $\mathrm{Q}^{*}$ was also not estimated. Finally, the risk of bias was not systematically addressed by QUADAS methodology. The findings of the present meta-analysis when combined with the study of Monteiro et al argue in favor of using TCD as a standard ancillary test for brain death diagnosis, because TCD remains an inexpensive, easily repeatable, and noninvasive examination.

\section{Quality of Study}

Evaluation of QUADAS elicited several concerns about the quality of the studies that are largely unavoidable given the nonrandomized, observational nature of these individual studies. First, high bias was introduced in all studies regarding the risk of bias/index test. This was inevitable because TCD and any ancillary test will always be measured against and completed after clinical brain death confirmation. Regarding the risk of bias/patient selection, high bias was introduced in 4 studies and unclear bias was introduced in 4 studies. Azevedo et $\mathrm{al}^{27}$ introduced bias because they did not enroll patients in whom TCDs could not be obtained, while they failed to de- 


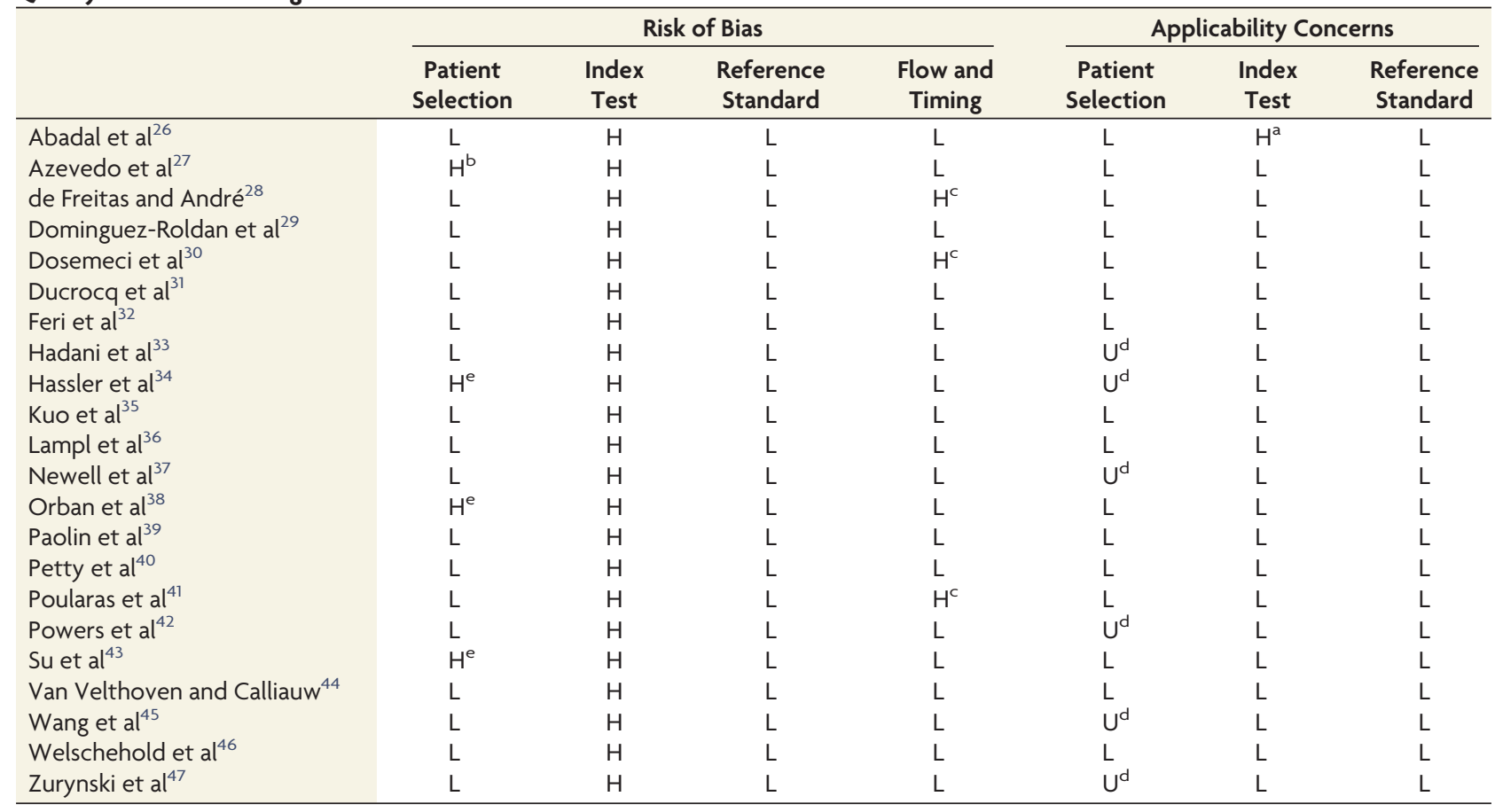

Note:- L indicates low-risk; $\mathrm{H}$, high-risk; $\mathrm{U}$, unclear.

a Used alternative TCD index tests: contrast-induced TCD.

${ }^{b}$ Did not enroll patients when TCD windows could not be obtained.

'Patients were excluded from analysis.

${ }^{\mathrm{d}}$ Subset of the population explored included children (younger than 18 years); not enough information provided to differentiate how many children were included in the study.

e Of the total number of patients clinically diagnosed with brain death, only a portion of these patients underwent TCD

Study
Abadal et al, 2008
Azevedo et al, 2000
De Freitas et al, 2006
Dominguez-Roldan et al, 1995
Dosemeci et al, 2004
Ducrocq et al, 1998
Feri et al, 1994
Hadani et al, 1999
Hassler et al, 1988
Kuo et al, 2006
Lampl et al, 2002
Newell et al, 1989
Orban et al, 2012
Paolin et al,1995
Petty et al, 1990
Poularas et al, 2006
Powers et al, 1989
Su et al, 2014
van Velthoven et al, 1988
Welschehold et al, 2012
Wang et al, 2008
Zurynski et al, 1991

$\begin{array}{rrrrr}\text { TP } & \text { FP } & \text { FN } & \text { TN } & \text { Sensitivity }(95 \% \text { Cl) } \\ 13 & 0 & 0 & 0 & 1.00[0.75,1.00] \\ 20 & 0 & 2 & 75 & 0.91[0.71,0.99] \\ 204 & 0 & 66 & 0 & 0.76[0.70,0.81] \\ 49 & 0 & 0 & 26 & 1.00[0.93,1.00] \\ 57 & 1 & 4 & 38 & 0.93[0.84,0.98] \\ 124 & 0 & 6 & 0 & 0.95[0.90,0.98] \\ 22 & 0 & 0 & 15 & 1.00[0.85,1.00] \\ 81 & 0 & 3 & 53 & 0.96[0.90,0.99] \\ 25 & 0 & 8 & 0 & 0.76[0.58,0.89] \\ 34 & 0 & 10 & 57 & 0.77[0.62,0.89] \\ 54 & 0 & 3 & 0 & 0.95[0.85,0.99] \\ 12 & 0 & 0 & 0 & 1.00[0.74,1.00] \\ 22 & 0 & 0 & 0 & 1.00[0.85,1.00] \\ 8 & 0 & 7 & 0 & 0.53[0.27,0.79] \\ 21 & 0 & 5 & 26 & 0.81[0.61,0.93] \\ 40 & 0 & 5 & 20 & 0.89[0.76,0.96] \\ 18 & 3 & 3 & 0 & 0.86[0.64,0.97] \\ 46 & 2 & 17 & 6 & 0.73[0.60,0.83] \\ 26 & 0 & 3 & 9 & 0.90[0.73,0.98] \\ 65 & 0 & 6 & 0 & 0.92[0.83,0.97] \\ 92 & 0 & 19 & 0 & 0.83[0.75,0.89] \\ 104 & 0 & 7 & 29 & 0.94[0.87,0.97]\end{array}$

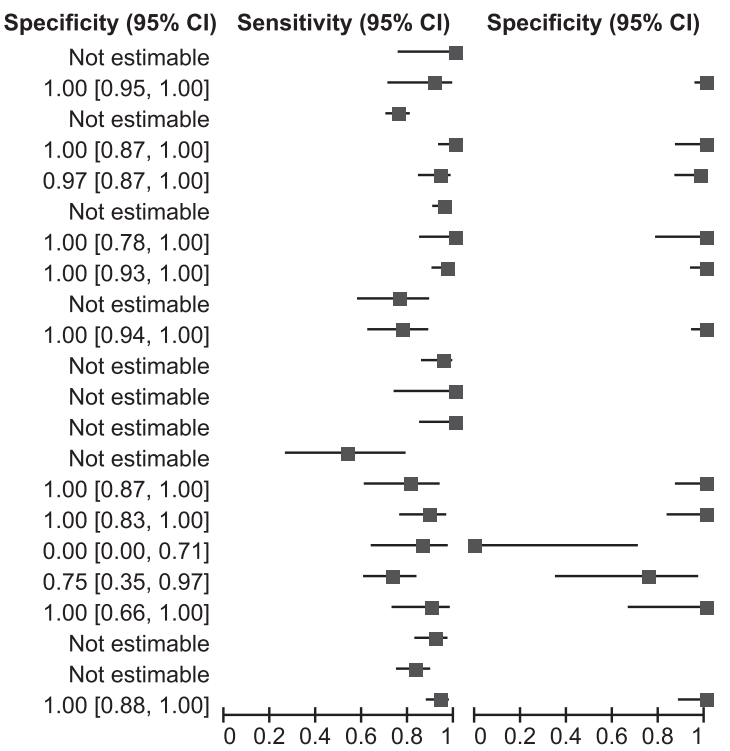

FIG 2. Sensitivity and specificity ratios of individual studies, with their corresponding $95 \%$ confidence intervals. TP indicates true-positive; FP, positive; FN, false-negative; TN, true-negative.

scribe the number of excluded patients. The other 3 articles introduced high bias in this category because they were primarily designed to use and compare various ancillary tests in brain death confirmation (TCD being used in addition to angiography, electroencephalography, intracranial pressure monitoring, and somatosensory-evoked potentials). ${ }^{34,38,43} \mathrm{Al}-$ though high bias was introduced in 3 studies regarding risk of bias/flow and timing, the exclusion of these patients was un- avoidable due to clinical instability. In all 3 studies, these patients were likely excluded because they had reached an advanced state of brain herniation that left them in multiorgan dysfunction with extreme hemodynamic instability. ${ }^{28,30,41}$ Unclear bias was introduced in 5 studies regarding applicability concerns/patient selection because pediatric populations were incorporated and analyzed. Enough details were not provided to individually remove the pediatric patients. Although 

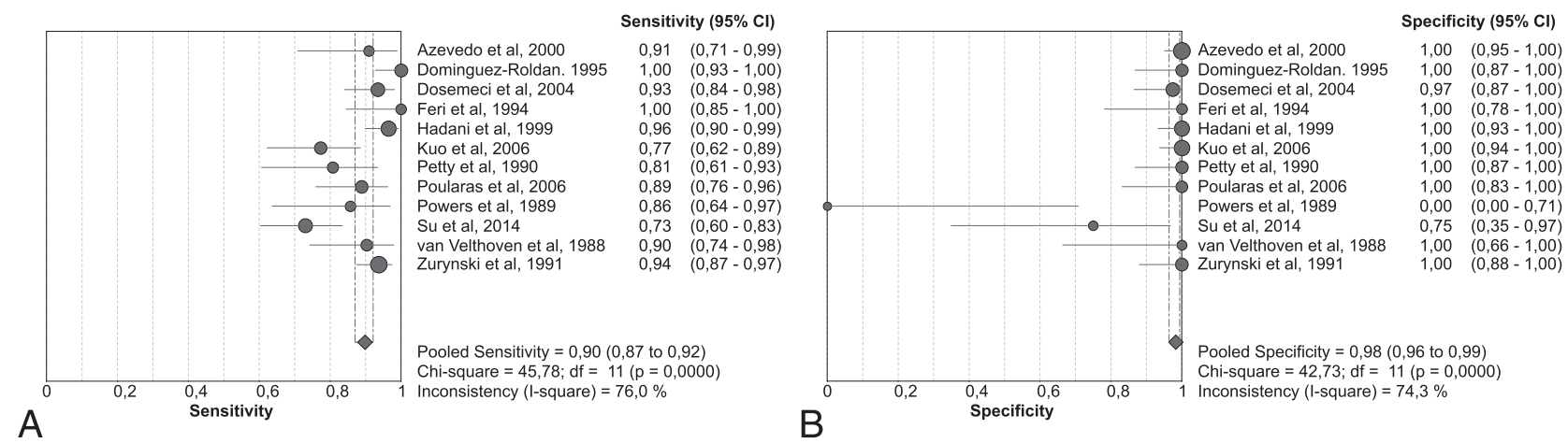

FIG 3. Pooled sensitivity $(A)$ and specificity $(B)$ of eligible studies reporting both diagnostic accuracy testing parameters.

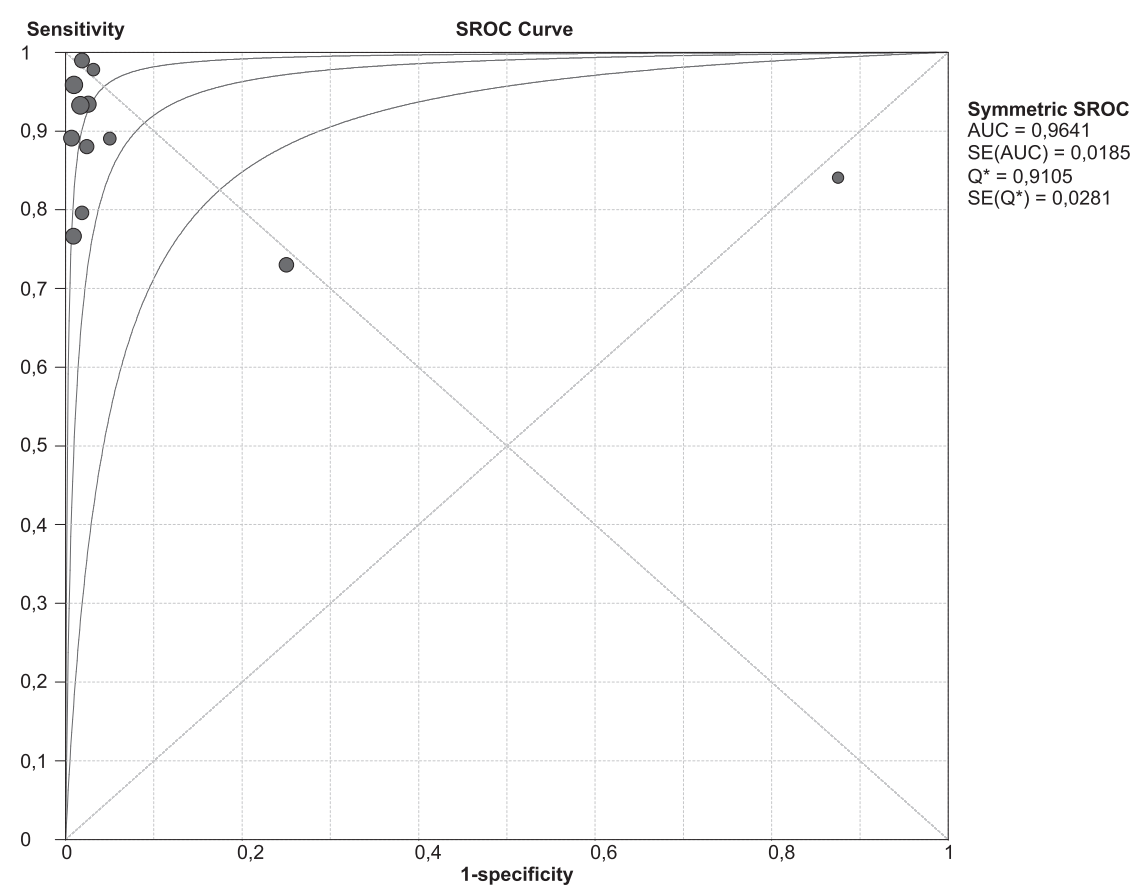

FIG 4. The summary receiver operating characteristic curve with its corresponding $95 \%$ confidence interval of the diagnostic threshold of transcranial Doppler in the diagnosis of brain death.

death, followed by CCA, and then a rebound improvement in the clinical examination (usually via resumption of respiratory drive), ${ }^{21}$ CCA represents a more severe pathologic state than brain death because it is characterized by refractory high intracranial pressures preventing cerebral perfusion. Therefore, CCA should usually temporally occur after brain stem dysfunction. This was shown because serial TCDs conducted on patients who were clinically brain dead always resulted in the best sensitivities occurring at later time points. ${ }^{30,33,38,41}$ In addition, the accuracy of TCD evaluation for brain death may also be subject to brain death criteria used in different countries, the largest difference being inclusion of apnea testing which was present in $59 \%$ of countries surveyed $^{48}$ and would likely result in lower TCD sensitivities.

Second, confirmation of brain death via TCD is influenced by the mechanism

unfortunate, as evidenced by mean ages, these populations were primarily adult. ${ }^{33,34,37,42,45,47}$ Finally, 1 study introduced high bias in applicability concerns/index test because while using tests that incorporated a standard TCD window (transtemporal), this study also introduced contrast-induced TCD to increase sensitivity. ${ }^{26}$

\section{Limitations}

Several limitations must be addressed when using TCDs for confirmation of brain death: 1) worse sensitivity of TCD compared with the clinical examination, 2) association between the mechanism of neurologic injury and the sensitivity of TCD, 3) the impossibility of having perfect sensitivity with TCDs, and 4) the impossibility of preventing false-positive results. First, TCD will ultimately have lower sensitivity than clinical confirmation of brain death at earlier time points because blood vessel velocities and patterns demonstrated on TCDs for brain death confirmation depend on CCA. Although isolated case reports exist demonstrating clinical confirmation of brain of neurologic injury. The classic neurologic injury leading to brain death is a supratentorial mass lesion with downward herniation into the brain stem. ${ }^{49}$ Because herniation continues due to increased intracranial pressure, cerebral perfusion will wane and finally be absent, resulting in CCA and corresponding TCD waveforms. Other etiologies that result in global cerebral edema (diffuse anoxic brain injury, leukoencephalopathy from toxin ingestion, poor-grade subarachnoid hemorrhage, and diffuse traumatic brain injury) will also lead to refractory intracranial pressures and CCA whose waveform patterns can be detected by TCD.

Third, TCDs will never offer perfect sensitivities because structural abnormalities will inevitably arise, primarily with obtaining adequate bone windows. Sixty-four patients were reported to have unobtainable bone windows during TCD examination in 8 studies of this meta-analysis (On-line Table), giving a corresponding insufficient insonation rate of $8.4 \%$ (range, $2.8 \%$ $16 \%)$. These patients were evaluated as false-negatives, and this decreased the sensitivity measure. In addition, due to unilateral supratentorial lesions, cases will occur in which 1 large vessel will 
demonstrate a CCA pattern on TCD without this occurring in the contralateral vessel. ${ }^{39}$ In addition, abnormalities of the cranium, primarily manifested by postsurgical procedures such as decompressive hemicraniectomy ${ }^{24,25}$ or external ventricular device placement, ${ }^{24}$ or the more malleable cranium of infants and young children, ${ }^{23}$ will lead to decreased sensitivities. The most likely mechanism for this was increased pulsatile arterial blood flow introduced by relief of intracranial pressure.

Fourth, although having an extremely high specificity, falsepositive results with TCDs are still possible and occurred in 3 studies. In Powers et al, ${ }^{42} 2$ of these false-positive cases occurred because though TCD confirmation did precede clinical brain death, unanticipated cardiopulmonary arrest prevented the inevitable confirmation of clinical brain death. The last false-positive result occurred in a 31-year-old woman with a gunshot wound to the head who had retrograde diastolic flow velocities noted on TCD but who also had extremely high net flow velocities. Her neurologic function improved, and she was ultimately discharged from the hospital. ${ }^{42}$ In Su et al, ${ }^{43} 2$ false-positives occurred; however, details behind these results were not evaluated in the article. Finally, Dosemeci et $\mathrm{al}^{30}$ had 1 false-positive brain death result with TCD, which was associated with a patient with TCD waveforms suggestive of CCA but who still had weak respiratory movements elicited by an apnea test.

Finally, no adjustment was reported in the individual study protocols, thus providing only the unadjusted sensitivity/specificity measures in the published reports of the included studies. Therefore pooling of unadjusted sensitivity/specificity measures can neither eliminate the risk of potential confounder existence in the included study protocols nor exclude their accession to the pooled estimates.

\section{Topics of Future Study}

Several topics may help clinicians better understand the limitations associated with TCD use in brain death confirmation. One such topic may be trying to better understand the mechanism and pathophysiology associated with CCA. This would include gaining a better understanding of the time lag that occurs from brain death to CCA. Such knowledge may assist in determining the earliest time to use TCD as ancillary confirmation of brain death. Other future studies would evaluate how TCD velocity changes relative to skull defects or abnormalities. Future studies would likely need to segregate infants and young children from adults because of their malleable skulls.

\section{CONCLUSIONS}

In our meta-analysis, TCD as ancillary testing for brain death was found to be highly sensitive and specific with rates of $89 \%$ and $98 \%$, respectively. However, the main limitation of TCD is that it detects CCA rather than brain stem dysfunction, which will inevitably result in less sensitivity than the clinical examination. As a result, TCD is still best suited for an ancillary test to be performed when clinical conditions or medications make the clinical examination unsuitable.

Disclosures: Georgios Tsivgoulis_UNRELATED: Grants/Grants Pending: I was supported by the European Regional Development Fund-Project St. Anne's University
Hospital, Brno International Clinical Research Center (FNUSA-ICRC) (No. CZ.1.05/1.1.00/02.0123)

\section{REFERENCES}

1. A definition of irreversible coma: Report of the Ad Hoc Committee of the Harvard Medical School to Examine the Definition of Brain Death. JAMA 1968;205:337-40 CrossRef Medline

2. Wijdicks EF. The diagnosis of brain death. N Engl J Med 2001;344: 1215-21 CrossRef Medline

3. Wijdicks EF, Varelas PN, Gronseth GS, et al; American Academy of Neurology. Evidence-based guideline update: determining brain death in adults: report of the Quality Standards Subcommittee of the American Academy of Neurology. Neurology 2010;74:1911-18 CrossRef Medline

4. Nakagawa TA, Ashwal S, Mathur M, et al; Society of Critical Care Medicine, Section on Critical Care and Section on Neurology of the American Academy of Pediatrics, Child Neurology Society. Guidelines for the determination of brain death in infants and children: an update of the 1987 Task Force recommendations. Crit Care Med 2011;39:2139-55 CrossRef Medline

5. Datar S, Fugate J, Rabinstein A, et al. Completing the apnea test: decline in complications. Neurocrit Care 2014;21:392-96 CrossRef Medline

6. Goudreau JL, Wijdicks EF, Emery SF. Complications during apnea testing in the determination of brain death: predisposing factors. Neurology 2000;55:1045-48 Medline

7. Bayliff CD, Schwartz ML, Hardy BG. Pharmacokinetics of high-dose pentobarbital in severe head trauma. Clin Pharmacol Ther 1985;38: 457-61 CrossRef Medline

8. Monteiro LM, Bollen CW, van Huffelen AC, et al. Transcranial Doppler ultrasonography to confirm brain death: a meta-analysis. Intensive Care Med 2006;32:1937-44 CrossRef Medline

9. Whiting PF, Rutjes AW, Westwood ME, et al; QUADAS-2 Group. QUADAS-2: a revised tool for the quality assessment of diagnostic accuracy studies. Ann Intern Med 2011;155:529-36 CrossRef Medline

10. Liberati A, Altman DG, Tetzlaff J, et al. The PRISMA statement for reporting systematic reviews and meta-analyses of studies that evaluate health care interventions: explanation and elaboration. J Clin Epidemiol 2009;62:e1-34 CrossRef Medline

11. Reitsma JB, Rutjes AW, Whiting P, et al. Chapter 9: assessing methodological quality. In: Deeks JJ, Bossuyt PM, Gatsonis C, eds. Cochrane Handbook for Systematic Reviews of Diagnostic Test Accuracy. The Cochrane Collaboration, 2009. http://srdta.cochrane.org/. Accessed September 30, 2014

12. Dominguez-Roldan JM, Garcia-Alfaro C, Jimenez-Gonzalez PI, et al. Brain death due to supratentorial masses: diagnosis using transcranial Doppler sonography. Transplant Proc 2004;36: 2898-900 CrossRef Medline

13. Sharma D, Souter MJ, Moore AE, et al. Clinical experience with transcranial Doppler ultrasonography as a confirmatory test for brain death: a retrospective analysis. Neurocrit Care 2011;14:370-76 CrossRef Medline

14. Shiogai T, Sato E, Tokitsu M, et al. Transcranial Doppler monitoring in severe brain damage: relationships between intracranial haemodynamics, brain dysfunction and outcome. Neurol Res 1990;12:205-13

15. Vakilian A, Iranmanesh F. Comparison of cerebral blood flow pattern by transcranial Doppler in patients with diffuse and focal causes of brain death. J Res Med Sci 2012;17:1156-60

16. Llompart-Pou JA, Abadal JM, Velasco J, et al. Contrast-enhanced transcranial color sonography in the diagnosis of cerebral circulatory arrest. Transplant Proc 2009;41:1466-68 CrossRef Medline

17. Kirkham FJ, Levin SD, Padayachee TS, et al. Transcranial pulsed Doppler ultrasound findings in brain stem death. J Neurol Neurosurg Psychiatry 1987;50:1504-13 CrossRef Medline

18. Qian SY, Fan XM, Yin HH. Transcranial Doppler assessment of brain death in children. Singapore Med J 1998;39:247-50 Medline 
19. Vicenzini E, Pulitano P, Cicchetti R, et al. Transcranial Doppler for brain death in infants: the role of the fontanelles. Eur Neurol 2010; 63:164-69 CrossRef Medline

20. Rodriguez RA, Cornel G, Alghofaili F, et al. Transcranial Doppler during suspected brain death in children: potential limitation in patients with cardiac "shunt." Pediatr Crit Care Med 2002;3:153-57 CrossRef Medline

21. Budohoski KP, Aries MJ, Kirkpatrick PJ, et al. Protracted cerebral circulatory arrest and cortical electrical silence coexisting with preserved respiratory drive and flexor motor response. $\mathrm{Br}$ J Anaest 2012;109:293-94 CrossRef Medline

22. Eder KE, Haussen DC, Searls DE, et al. Reverberating TCD flow pattern in brain death. Neurology 2012;79:e79 CrossRef Medline

23. Mata-Zubillaga D, Oulego-Erroz I. Persistent cerebral blood flow by transcranial Doppler ultrasonography in an asphyxiated newborn meeting brain death diagnosis: case report and review of the literature. J Perinatal 2012;32:473-75 CrossRef Medline

24. Thompson BB, Wendell LC, Potter NS, et al. The use of transcranial Doppler ultrasound in confirming brain death in the setting of skull defects and extraventricular drains. Neurocrit Care 2014;21: 534-38 CrossRef Medline

25. Cabrer C, Dominguez-Roldan JM, Manyalich M, et al. Persistence of intracranial diastolic flow in transcranial Doppler sonography exploration of patients in brain death. Transplant Proc 2003;35: 1642-43 CrossRef Medline

26. Abadal JM, Llompart-Pou JA, Homar J, et al. Ultrasonographic cerebral perfusion in assessment of brain death: a preliminary study. J Ultrasound Med 2008;27:791-94 Medline

27. Azevedo E, Teixeira J, Neves JC, et al. Transcranial Doppler and brain death. Transplant Proc 2000;32:2579-81 CrossRef Medline

28. de Freitas GR, André C. Sensitivity of transcranial Doppler for confirming brain death: a prospective study of 270 cases. Acta Neurol Scand 2006;113:426-32 CrossRef Medline

29. Dominguez-Roldan JM, Murillo-Cabezas F, Muñoz-Sanchez A, et al. Changes in the Doppler waveform of intracranial arteries in patients with brain-death status. Transplant Proc 1995;27:2391-92 Medline

30. Dosemeci L, Dora B, Yilmaz M, et al. Utility of transcranial Doppler ultrasonography for confirmatory diagnosis of brain death: two sides of the coin. Transplantation 2004;77:71-75 CrossRef Medline

31. Ducrocq X, Braun M, Debouverie $M$, et al. Brain death and transcranial Doppler: experience in 130 cases of brain dead patients. J Neurol Sci 1998;160:41-46 CrossRef Medline

32. Feri M, Ralli L, Felici M, et al. Transcranial Doppler and brain death diagnosis. Crit Care Med 1994;22:1120-26 CrossRef Medline

33. Hadani M, Bruk B, Ram Z, et al. Application of transcranial Doppler ultrasonography for the diagnosis of brain death. Intensive Care Med 1999;25:822-28 CrossRef Medline

34. Hassler W, Steinmetz H, Gawlowski J. Transcranial Doppler ultra- sonography in raised intracranial pressure and in intracranial circulatory arrest. J Neurosurg 1988;68:745-51 Medline

35. Kuo JR, Chen CF, Chio CC, et al. Time dependent validity in the diagnosis of brain death using transcranial Doppler sonography. J Neurol Neurosurg Psychiatry 2006;77:646-49 CrossRef Medline

36. Lampl Y, Gilad R, Eschel Y, et al. Diagnosing brain death using the transcranial Doppler with a transorbital approach. Arch Neurol 2002;59:58-60 CrossRef Medline

37. Newell DW, Grady MS, Sirotta P, et al. Evaluation of brain death using transcranial Doppler. Neurosurgery 1989;24:509-13 CrossRef Medline

38. Orban JC, El-Mahjoub A, Rami L, et al. Transcranial Doppler shortens the time between clinical brain death and angiographic confirmation: a randomized trial. Transplantation 2012;94:585-88 CrossRef Medline

39. Paolin A, Manuali A, Di Paola F, et al. Reliability in diagnosis of brain death. Intensive Care Med 1995;21:657-62 CrossRef Medline

40. Petty GW, Mohr JP, Pedley TA, et al. The role of transcranial Doppler in confirming brain death: sensitivity, specificity, and suggestions for performance and interpretation. Neurology 1990;40: 300-03 CrossRef Medline

41. Poularas J, Karakitsos D, Kouraklis G, et al. Comparison between transcranial color Doppler ultrasonography and angiography in the confirmation of brain death. Transplant Proc 2006;38:1213-17 CrossRef Medline

42. Powers AD, Graeber MC, Smith RR. Transcranial Doppler ultrasonography in the determination of brain death. Neurosurgery 1989 ; 24:884-89 CrossRef Medline

43. Su Y, Yang Q, Liu G, et al. Diagnosis of brain death: confirmatory tests after clinical test. Chin Med J (Engl) 2014;127:1272-77 Medline

44. Van Velthoven V, Calliauw L. Diagnosis of brain death: transcranial Doppler sonography as an additional method. Acta Neurochir (Wien) 1988;95:57-60 CrossRef Medline

45. Wang K, Yuan Y, Xu ZQ, et al. Benefits of combination of electroencephalography, short latency somatosensory evoked potentials, and transcranial Doppler techniques for confirming brain death. J Zhejiang Univ Sci B 2008;9:916-20 CrossRef Medline

46. Welschehold S, Boor S, Reuland K, et al. Technical aids in the diagnosis of brain death: a comparison of SEP, AEP, EEG, TCD and CT angiography. Dtsch Arztebl Int 2012;109:624-30 CrossRef Medline

47. Zurynski Y, Dorsch N, Pearson I, et al. Transcranial Doppler ultrasound in brain death: experience in $\mathbf{1 4 0}$ patients. Neurol Res 1991; 13:248-52 Medline

48. Wijdicks EF. Brain death worldwide: accepted fact but no global consensus in diagnostic criteria. Neurology 2002;58:20-25 CrossRef Medline

49. Ropper AH. A preliminary MRI study of the geometry of brain displacement and level of consciousness with acute intracranial masses. Neurology 1989;39:622-27 CrossRef Medline 